\title{
Rapport annuel sur les activités de la FMH dans le domaine des assistantes médicales
}

\section{Adrian Sury}

Président des délégués cantonaux aux questions des assistantes médicales
Correspondance: Dr Adrian Sury Elfenstr. 18 CH-3000 Berne 15 Tél. 0313591111 Fax 0313591112
Un nouveau changement d'ordre organisationnel s'est produit au cours de la période passée sous revue. Après de nombreuses années de travail intensif en faveur de la formation des assistantes médicales, le Dr Peter Tschudi s'est retiré de la présidence de la Commission suisse pour le développement professionnel et la qualité. Nous le remercions très cordialement pour son important engagement, notamment dans l'élaboration de la nouvelle ordonnance sur la formation et du plan de formation ainsi que pour leur mise en œuvre. Nous avons trouvé son successeur en la personne du Dr Thomas Heuberger, élu lors de la séance de commission d'avril 2013. En sa qualité de président de l'Organisation du monde du travail (ORTRA) Formation professionnelle des assistantes médicales, en charge de la formation supérieure des assistantes médicales, il assurera le lien avec la formation initiale.

Le Dr Ernst Gähler, vice-président de la FMH et responsable du domaine Professions paramédicales, continue de représenter les différents organes au Comité central de la FMH. La répartition des tâches est désormais la suivante:

- Délégués aux questions des assistantes médicales: Dr A. Sury, président, et Dr R. Tognina, vice-président

- Commission suisse pour le développement professionnel et la qualité: Dr Th. Heuberger, président

- Commission d'examen pour la procédure de qualification des assistantes médicales: Dr K. Hubschmid, présidente

- ORTRA Formation professionnelle des assistantes médicales: Dr Th. Heuberger, président

La circulation des informations entre les différents acteurs, leurs domaines d'activité et le Comité central est très bien gérée par Elisabeth Tröhler, du service Assistantes médicales de la FMH. Le Bureau Assistantes médicales, composé des responsables susmentionnés, se réunit généralement une fois par an ou lors de séances spéciales pour discuter des problèmes, recoupements ou projets éventuels.

Au cours de la période passée sous revue, les procédures de qualification (anciennement examens de fin d'apprentissage) se sont déroulées pour la première fois selon la nouvelle ordonnance sur la formation (cf. rapport du Dr K. Hubschmid). Les experts ont dû suivre des cours portant sur les nouveautés telles que les notes attribuées dans le cadre des relations avec les patients ou de l'hygiène. Après quelques difficultés et problèmes de communication avec l'IFFP, les cours organisés pour les experts se sont déroulés avec succès grâce au Dr K. Hubschmid et aux responsables des groupes d'auteurs de la Commission d'examen.

La Commission pour le développement professionnel et la qualité s'est réunie deux fois au cours de la période passée sous revue (cf. rapport du Dr Th. Heuberger).

Le domaine de la formation supérieure connaît également une évolution réjouissante. L'ORTRA Formation professionnelle des assistantes médicales a déposé une demande auprès du SEFRI (anciennement OFFT) en vue d'un examen professionnel donnant lieu à un brevet fédéral (cf. rapport du Dr Th. Heuberger).

\section{Rapport de la Commission suisse pour le développement professionnel et la qualité Th. Heuberger}

La Commission pour le développement professionnel et la qualité a tenu deux séances au cours de la période passée sous revue. Pour l'essentiel, elle a examiné des questions relatives à l'application de la nouvelle ordonnance sur la formation (par ex. uniformisation des bulletins de notes, cours de formation pour experts, directives pour la procédure de qualification). Elle s'est penchée en outre sur une réglementation/recommandation pour la formation initiale complémentaire à l'extérieur du cabinet médical lorsque celui-ci ne dispose pas d'un laboratoire et/ou d'une installation de radiologie. Il est prévu d'intégrer cette recommandation dans le contrat d'apprentissage.

Les membres de la Commission ont d'ores et déjà discuté de quelques points faibles de l'ordonnance sur la formation et du plan de formation, lesquels devront être révisés à la prochaine occasion.

Comme le Dr K. Hubschmid siège à la Commission pour le développement professionnel et la qualité en sa qualité de présidente de la Commission d'examen, le lien avec la procédure de qualification et les demandes de révision de la Commission d'examen est garanti.

Entre-temps, les documents pour la validation des compétences de candidats provenant d'autres hori- 
zons professionnels ont été mis au point. Après leur adoption par la Commission, ces documents devront encore être approuvés par le SEFRI.

\section{Formation supérieure des assistantes médicales - Rapport de l'ORTRA Formation professionnelle des assistantes médicales Thomas Heuberger}

La période passée sous revue a été marquée essentiellement par les travaux consacrés au développement du profil professionnel en vue du projet Examen professionnel Coordinatrice médicale en cabinet sanctionné par un brevet fédéral ainsi qu'aux négociations visant à clarifier la position des assistantes médicales par rapport à d'autres professions de la santé et aux discussions de délimitation avec d'autres groupes professionnels intéressés.

Ce projet a nécessité plusieurs séances de même qu'un grand nombre de communications écrites pour éclaircir les ambiguïtés et déterminer la procédure d'envoi au SEFRI. Les contacts soutenus avec les autres organisations principales des professions de la santé ont finalement permis d'organiser un séminaire d'une journée et de les y inviter pour affiner le projet et délimiter les risques de conflit. Ce séminaire a eu lieu le 2 avril 2013 au siège de la be-med AG à l'Alpeneggstrasse à Berne sous la direction de Madame C. Gasser, OFSP; la conduite de la séance a été assumée par Monsieur M. Stalder.

Les objectifs, les intentions et les documents de base ont obtenu un écho positif; la nécessité et le sens d'un tel développement pour cette profession ont été reconnus sur le fond, tout spécialement en raison de l'importance que ce projet revêt pour l'avenir des cabinets de médecine de premier recours et de manière générale pour le travail au cabinet médical. Cela se reflète notamment dans les exigences formulées par l'initiative pour la médecine de famille ainsi que dans l'intérêt manifesté par l'OFSP dans le cadre de l'élaboration du plan directeur du DFI.

Les nombreux contacts avec des représentants de l'ASI, de la Ligue pulmonaire, de Médecins de famille Suisse, de l'ORTRASanté, des associations de soins à domicile, des assistantes médicales (SVA, ARAM) et des nutritionnistes ont permis d'éclaircir les malentendus dans de nombreux domaines, de déterminer les conflits éventuels dans la délimitation des compétences et de reformuler les dispositions réglementaires et les idées relatives au profil professionnel des assistantes médicales. Les modules définis dans le projet en ont été affectés et ont subi plusieurs adaptations.

Une fois ces idées et remarques prises en compte, le projet a été envoyé aux associations professionnelles intéressées pour une nouvelle consultation, actuellement en cours.

La Fédération suisse des associations d'assistantes médicales a été une nouvelle fois informée du projet lors d'une séance séparée au cours de laquelle elle a exprimé ses réflexions et ses doutes. Elle a déclaré ne pas vouloir s'opposer au projet sous cette forme mais pas non plus le soutenir car elle a l'intention de développer un projet analogue de son côté.

Il nous faudra probablement organiser une deuxième séance pour les dernières mises au point avant d'envoyer le projet au SEFRI (en novembre 2013 selon la planification) en espérant que le projet définitif pourra être mis en œuvre au printemps 2014 (après approbation et traduction).

Les contacts intensifs avec l'OFSP (Madame C. Gasser) ont conduit à de nouveaux éclaircissements; ils ont également permis d'améliorer les formulations et d'adapter le projet et la procédure. L'OFSP s'intéresse donc vivement au projet (cf. plan directeur du DFI) et nous sommes très reconnaissants du soutien et des conseils accordés par Madame Gasser.

Une discussion de fond est prévue prochainement avec Madame Aeby du SEFRI pour mener le projet activement sur la voie de son approbation; on espère atteindre rapidement la phase opérationnelle afin de pouvoir répondre aux demandes des écoles et des nombreuses assistantes médicales qui se renseignent sur la situation actuelle, les perspectives de la formation et sa reconnaissance officielle.

\section{La période passée sous revue a été marquée essentiellement par les travaux consacrés au dévelop- pement du profil professionnel.}

Des séances ont été tenues pour identifier les modules, en fixer la valeur, discuter des conditions et définir l'importance de chaque domaine en particulier (caractère facultatif ou obligatoire pour la reconnaissance des deux orientations prévues: clinique et administrative).

Perspectives:

Nous espérons que le projet pourra être envoyé au SEFRI à fin 2013 et que la procédure de reconnaissance sera achevée au printemps 2014. Ensuite, les écoles pourront préparer leurs offres de formation, à moins que certaines institutions ne l'aient déjà fait, compte tenu de nos informations. Autrement dit, les premiers cursus pourraient commencer en été 2014.

La question du financement du projet ORTRA Formation professionnelle des assistantes médicales reste ouverte. On envisage la création d'un fonds, ou éventuellement d'autres modèles de financement. Des résultats sont également attendus en 2014 car le projet de formation devra être développé, complété puis mis à jour régulièrement. 


\section{Commission d'examen pour la procédure de qualification des assistantes médicales Katrin Hubschmid}

Au cours de l'année passée sous revue, la Commission a élaboré les documents d'examen pour la première fois selon la nouvelle ordonnance sur la formation, ce qui a constitué un véritable défi pour tous les groupes d'auteurs. Nous exprimons à leurs membres nos vifs remerciements pour leur engagement hors du commun et grâce auquel les documents ont pu être livrés dans les délais.

\section{La demande de places d'apprentissage et l'intérêt pour cette profession restent élevés.}

Après un début très difficile, les cours nécessaires pour les experts d'examen se sont déroulés sans problème et ont pu avoir lieu avant la procédure de qualification.

Pour les écoles, les enseignants et les experts, l'organisation de l'examen a représenté une charge extraordinaire, car il a fallu d'une part examiner de nouvelles branches et d'autre part mettre au point de nouveaux examens écrits.

A notre grande satisfaction, la procédure de qualification s'est déroulée partout conformément aux directives. Nous remercions cordialement tous les participants.
Comme nous le craignions, la mise en œuvre de la nouvelle ordonnance sur les examens a donné lieu à des difficultés, comme en témoignent les nombreux retours reçus. Avant tout, l'évaluation des nouveaux objectifs généraux «Relations avec les patients» et «Hygiène», mais en partie aussi l'objectif général «Processus d'entreprise» dans le cadre des travaux pratiques est fortement mise en question, car elle masque de graves lacunes dans les compétences de base des assistantes médicales («Diagnostic de laboratoire», «Diagnostic radiologique», «Diagnostic général, processus thérapeutiques, assistance médicale, activités de conseil» [anciennement «Assistance à la consultation»] et «Bases médicales») En outre, le fait que cela ne ressorte pas du certificat fédéral de capacité (CFC) est insatisfaisant.

Il reste encore beaucoup à faire pour atteindre l'objectif visé, à savoir des examens fiables, évaluables et objectifs.

\section{Formation de base des assistantes médicales et cours de maîtres d'apprentissage}

Sur les 895 personnes en formation qui ont passé l'examen cette année, 847 l'ont réussi.

Ainsi que le montre l'enquête menée chaque année auprès des écoles formant des assistantes médicales, on devrait pouvoir compter ces prochaines années avec le même nombre d'assistantes diplômées. 812 personnes se trouvent actuellement en $3^{\mathrm{e}}$ année d'apprentissage, 857 en $2^{\mathrm{e}}$ année et 881 candidates ont commencé leur formation cette année. En 2013, 124 d'entre elles ont choisi une école privée alors qu'elles étaient env. 90 en 2012 et autant en 2011.

Figure 1

Statistique des assistantes médicales diplômées depuis 1992.

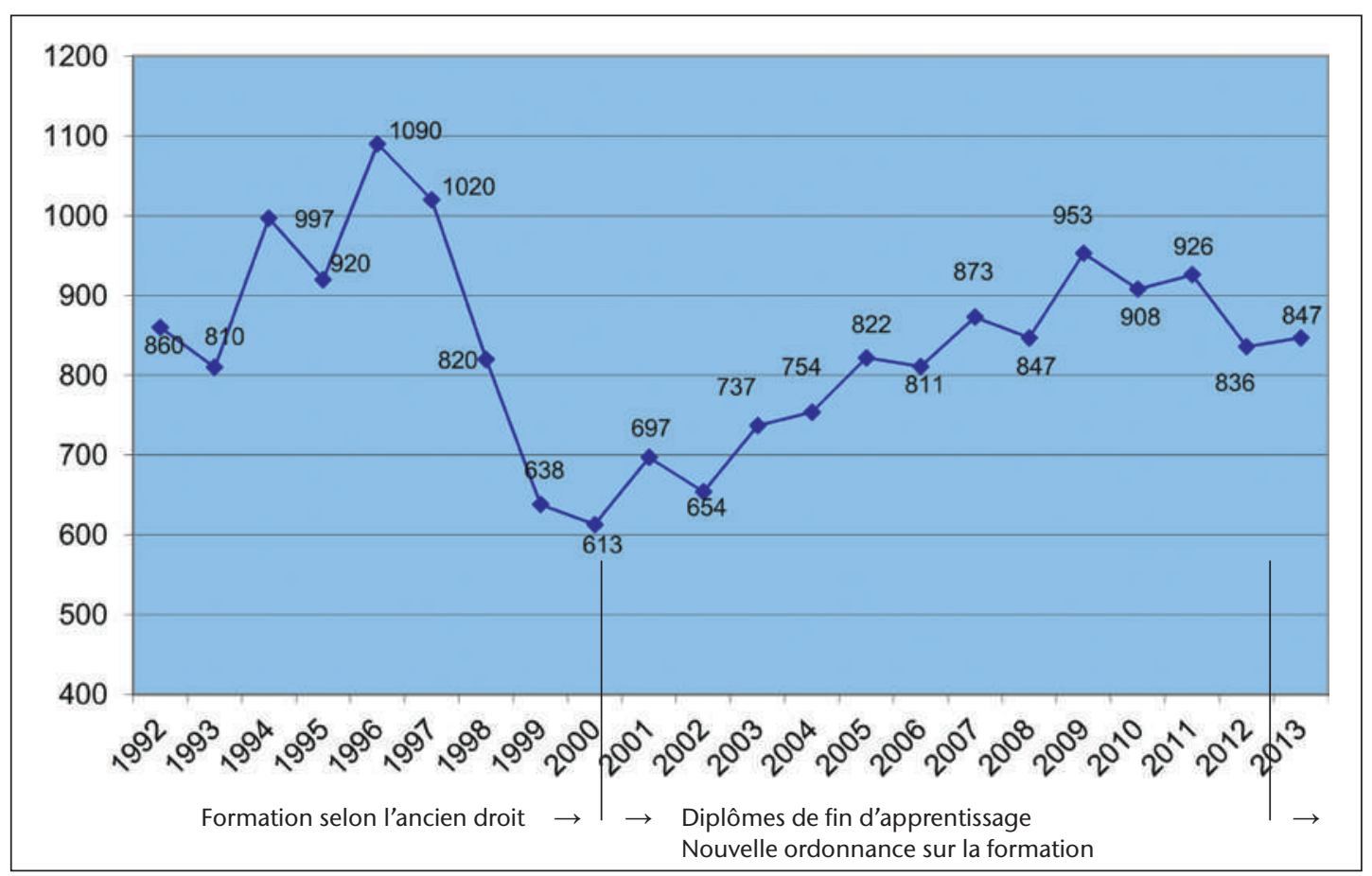


La demande de places d'apprentissage et l'intérêt pour cette profession restent donc élevés. Pour qu'un nombre suffisant d'assistantes médicales puisse être formé à l'avenir également, il est indispensable de continuer à offrir des places d'apprentissage en conséquence. A cet égard, il est important de signaler que la possibilité d'obtenir une autorisation de maître d'apprentissage auprès de l'office cantonal de la formation professionnelle n'est pas uniquement réservée aux médecins de premier recours disposant d'un laboratoire et d'une installation de radiologie; elle est également ouverte à d'autres spécialistes ainsi qu'aux hôpitaux et cliniques offrant des prestations ambulatoires.

\section{Cours de maîtres d'apprentissage pour les médecins}

Durant la période passée sous revue, la FMH a de nouveau organisé deux cours de maîtres d'apprentissage en collaboration avec les écoles Juventus-Woodtli à Zurich, comme elle le fait depuis de nombreuses années. des informations concernant l'engagement d'assistantes médicales diplômées (modèle de contrat de travail, recommandations salariales, protection de la maternité).

\section{Office de conciliation}

Durant la période passée sous revue, trois demandes de conciliation pour la Suisse allemande sont parvenues au Prof. J. Brühwiler, docteur en droit. Ces cas sont encore en suspens.

\section{Service Assistantes médicales du Secrétariat général}

La section Assistantes médicales du Secrétariat général de la FMH est au service du président des délégués aux questions des assistantes médicales ainsi que du Dr Ernst Gähler pour toutes les affaires concernant les assistantes médicales. Il fonctionne comme plaque tournante entre tous les organes cités ci-avant. En outre, il gère les secrétariats de la Commission d'examen pour la procédure de qualification des assistantes médicales et de la Commission suisse pour le

\section{Le site internet www.am-suisse.ch propose des informations et des documents utiles.}

L'ancien règlement de formation exigeait de chaque détenteur de cabinet désireux d'obtenir une autorisation de maitre d'apprentissage de suivre un cours de formateur. Avec la nouvelle ordonnance sur la formation, il revient maintenant à l'assistante médicale de suivre un cours de maître d'apprentissage, comme l'exigent différents cantons de manière explicite. Pour cette raison et parce que, de surcroît, l'autorisation d'un programme de cours allégé pour les médecins a été remise en question, le Bureau Assistantes médicales a décidé de ne plus offrir de cours à l'avenir.

Les assistantes médicales qui aimeraient continuer à se former dans ce domaine pourront suivre les cours organisés par l'Association suisse des assistantes médicales (SVA). En outre, certains cantons proposent également des cours de maîtres d'apprentissage.

\section{www.am-suisse.ch - www.fmh.ch}

Le site internet www.mpaschweiz.ch, qui propose des informations et des documents utiles en lien avec la formation professionnelle initiale des assistantes médicales, est régulièrement mis à jour par le secrétariat des assistantes médicales de la FMH. Le site en français est désormais accessible à l'adresse www.am-suisse.ch.

A l'adresse www.fmh.ch $\rightarrow$ Services $\rightarrow$ Assistantes médicales se trouvent des documents types et développement professionnel et la qualité. Ce service est rattaché au Service juridique de la FMH, ce qui garantit la mise à jour régulière des règlements, conventions, etc., ainsi que les renseignements juridiques donnés aux médecins dans le domaine du droit du travail.

\section{En conclusion}

Comme à l'accoutumée, je remercie cette année également toutes les personnes qui se sont engagées avec détermination en faveur des assistantes médicales. Ma gratitude s'adresse aussi à toutes les assistantes médicales formatrices, aux maîtres d'apprentissage, aux écoles professionnelles et aux associations d'assistantes médicales. J'aimerais remercier tout particulièrement Barbara Linder et Elisabeth Tröhler pour leur importante contribution et les compétences dont elles font preuve dans notre service de coordination au Secrétariat général de la FMH ainsi que pour leur disponibilité. Au printemps, Elisabeth Tröhler a repris la direction administrative du domaine Professions paramédicales et donc particulièrement de la formation professionnelle des assistantes médicales; elle remplace Barbara Linder qui quitte cette fonction après l'avoir occupée pendant 13 ans afin de se consacrer entièrement à la suppléance du directeur de l'Institut suisse pour la formation médicale postgraduée et continue (ISFM). 